\title{
Intraspecific variability in herbivore performance and host quality: a field study of Uroleucon caligatum (Homoptera: Aphididae) and its Solidago hosts (Asteraceae)
}

NANCY MORAN Division of Biological Sciences and Museum of Zoology, University of Michigan, Ann Arbor, Michigan

ABSTRACT. 1. Performances of two clones of Uroleucon caligatum on eleven clones of Solidago were measured by caging aphids on plants in the field.

2. Several measures were obtained, including developmental time from birth to adulthood, size of first instar nymphs, adult weight, and total colony weight.

3. All measures of performance were strongly affected by plant clone.

4. Effect of aphid clone-plant clone interaction was significant only for first instar size.

5. In a subsequent screenhouse experiment, plant clones were subjected to uniform conditions and still exhibited large differences in host quality.

\section{Introduction}

Few studies of insect-plant relationships have focused on naturally occurring intraspecific variability either in host quality or in herbivore efficiency (but see Edmunds \& Alstad, 1978; Mitter et al., 1979; Journet, 1980). Such variation is regularly documented in crop plants and the insects which feed upon them (reviews in Day, 1974; Gallun et al., 1975). Particularly well studied are differences between crop varieties in resistance to aphids and differences between aphid strains (biotypes) in colonizing ability (summarized by Eastop, 1973). The extent of such variation within and between natural populations is central to theories concerning host-parasite coevolution, the advantages of sexuality (Hamilton, 1980), and the maintenance of genetic variability within populations (Clarke, 1976).

Aphids colonizing perennial herbs are well suited for studies aimed at discovering the extent of this variation because they permit replicate measurements of each herbivore genotype-host genotype combination. (Most

Correspondence: Nancy Moran, Division of Biological Sciences and Museum of Zoology, University of Michigan, Ann Arbor, Michigan 48109, U.S.A. evidence, as summarized by Blackman (1979), supports genetic identity of successive, allfemale generations of aphids.) Uroleucon caligatum (Richards), the aphid species I studied, is parthenogenetic from May to September and spends its entire life cycle on goldenrods (Solidago spp.), primarily colonizing species of the Solidago canadensis $\mathrm{L}$. species complex, although it is sometimes found on other goldenrod species (Richards, 1972, Pielou, 1974; personal observations). Both wingless and winged morphs are produced throughout the summer, the latter being primarily responsible for initiating new colonies on previously aphid-free host clones.

In the study described in this paper, I tested for both intra- and interspecific differences in host plant quality through comparison of aphid performance on clones of the same and different species of Solidago. In addition, the possibility of intraspecific variation of the aphid was examined by comparing clones of caligatum.

\section{Materials and Methods}

Aphid clones. Each of the two aphid clones in the study originated from a single parthenogenetic female collected in May 1979. One of 
the females was taken from Solidago altissima L. in Allegan County, Michigan (AC clone), the other from S.altissima on the E. S. George Reserve (ESGR), Livingston County, Michigan (CP clone). Cultures of each clone were maintained in a screenhouse on the ESGR, in whole plant cages on potted stems of S.altissima.

Plant clones. Two clones each of S.altissima and S.gigantea Ait. and seven clones of S.canadensis were tested. The species used are perennials and closely related; canadensis and altissima are sometimes considered varieties of a single species (Gleason \& Cronquist, 1963). All grew on the ESGR in a field area selected to maximize homogeneity of microhabitat. The site lacked tall shrubs or trees; associated plants were mainly tall herbs including Aster umbellatus Mill., Apocynum cannabinum L., Pycnanthemum virginianum (L.) Durand \& Jackson and Urtica gracilis Ait. Clones of canadensis and gigantea (which produce relatively short rhizomes at this site) were distinguished through separation in space and also by unearthing and checking rhizomatous connections when the experiment finished. Stems of the two altissima clones were completely intermixed and were indentified on the basis of differences in leaf shape, venation and pubescence.

Measures of performance. Aphid performance was measured by caging aphids on plants in the field. Cages were constructed by cutting $40 \mathrm{~mm}$ diameter holes in the tops and bottoms of $50 \mathrm{~mm}$ plastic Petri dishes, covering the holes with 32-mesh Lumite screening (too small for aphid passage), and gluing foam rubber to the rims of the dishes (to seal gaps between cages and plants). The two halves of each cage were held in place with plant ties and supported by stakes. Uroleucon species produce little honeydew, so honeydew accumulation was not a problem. U.caligatum fed readily in the cages. Cages were placed one per stem near the apices where the aphids normally feed; during the experiments, cage position was adjusted as necessary to compensate for plant grow th.

Measurements of aphid performance were obtained as follows: On 4-5 August 1979, individual apterae from the stock colonies were confined overnight on the test plants and then removed together with any nymphs in excess of three per cage. Cages of the two aphid clones were in termixed at random within plant clones. The cages were checked every 2 days and date of moulting to adulthood recorded. These colonies were allowed to multiply until 29 August, 367 day-degrees after cage initiation, when all cages and contents were removed. (Day-degrees were calculated by averaging the daily minimum and maximum temperatures and subtracting $4.4^{\circ} \mathrm{C}$, the approximate theshold temperature for the development of U.caligatum, as determined in a growth chamber.) Fresh weights were obtained for the whole caged colonies and (some plant clones only) for three to five apterae per cage. A total of 707 first instar nymphs, three to six per cage, were mounted on microscope slides and their hind tibiae measured. Even for the fastest-growing colonies, the experiment was terminated before crowding was sufficient to induce production of winged morphs; thus there was no possibility of morph-specific differences in growth and reproduction affecting relative colony performance.

For each aphid clone-plant clone combination, ten cages were initiated; however, thirteen of the 220 cages were lost, mainly due to predators getting into cages.

In 1980, four of the canadensis clones used in 1979 field experiments were transplanted to pots and grown in a screenhouse. Transplants were dug in early April and consisted of undeveloped shoots plus $6 \mathrm{~cm}$ of lateral rhizome. Apterae from a single caligatum clone were used to initiate caged colonies on ten stems of each of the four plant clones. Colonies were allowed to develop from 2 August until 20 August (311 day-degrees) when cages where removed and fresh adult weights obtained.

To determine whether aphid performance was affected by plant clone, aphid clone, or an interaction between aphid and plant clones. two-way analysis of variance was performed on mean adult weight per cage, natural log of total colony weight, and mean first instar hind tibial length per cage. Log transformation of colony weight was necessary to satisfy assumption of normality. Loss of some cages resulted in unequal sample sizes. In order to simplify analysis and use as much of the data as possible, two-way anova for unequal but 
proportional sample sizes was used (Sokal \& Rohlf, 1969). This procedure required that values for seven cages be discarded, so that the two aphid clones had the same number of values within each plant clone. Non-parametric statistics were used for developmental times, as these data did not meet assumptions of analysis of variance.

\section{Results}

For all four measures of aphid performance, effect of plant clone is highly significant; however, significant aphid clone or interaction effects are found only for the measurement of first instar hind tibial lengths (Fig. 1). Effects of plant clone are highly significant whether

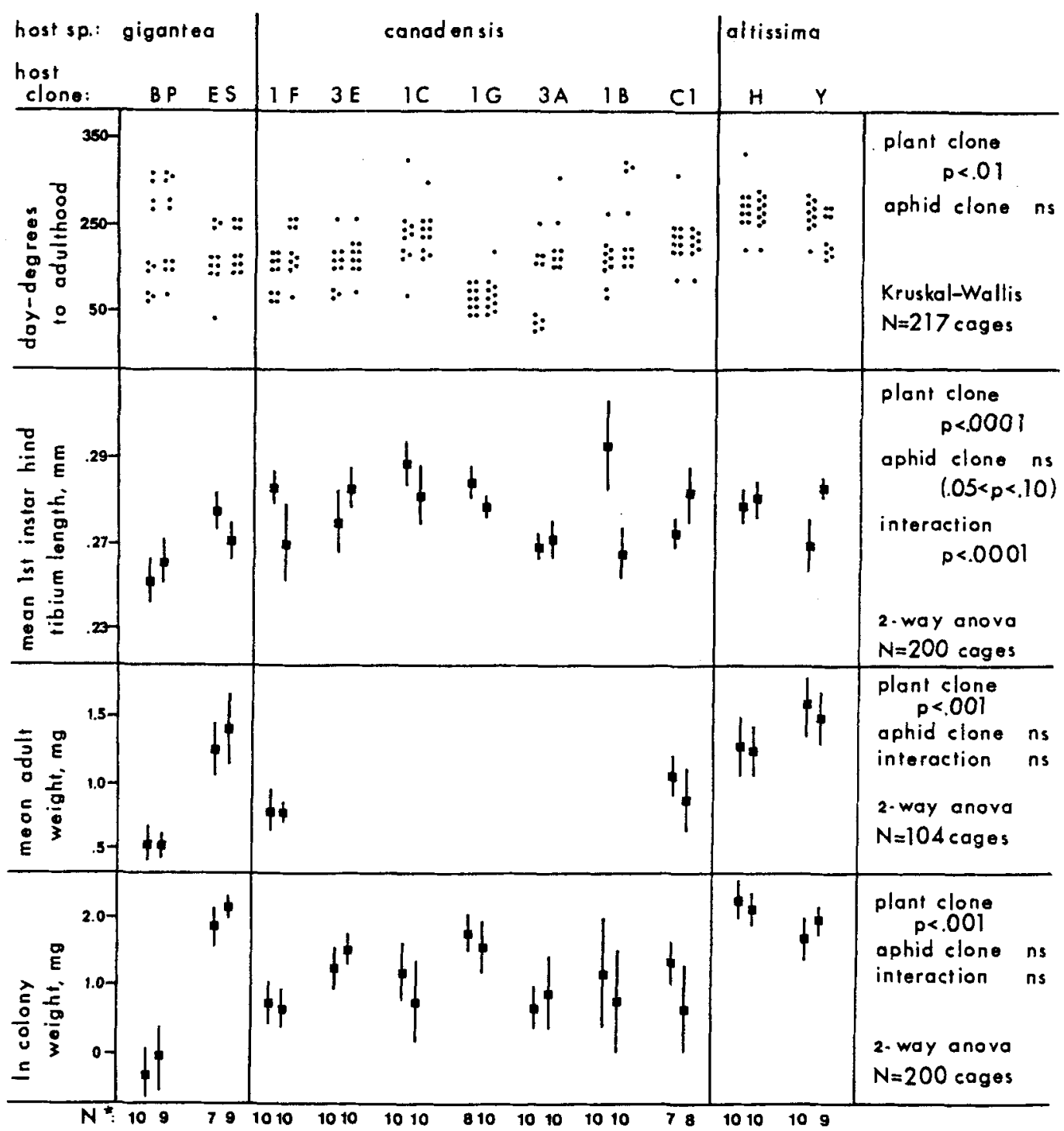

FIG. 1. Performance of two clones of Uroleucon caligatum on eleven clones of Solidago, as measured in the field. In each host clone column, caligatum clone $A C$ is on the left, CP on the right. (a) Developmental times, each dot is one cage. (b) Cage means of first instar hind tibial lengths. (c) Cage means of adult weights. (d) Natural logs of total weights of all aphids in each cage. Means and $95 \%$ confidence intervals are shown for (b)-(d). - Samples sizes for (b)-(d) are sometimes smaller than for (a) due to loss of cages subsequent to aphids reaching adulthood. 

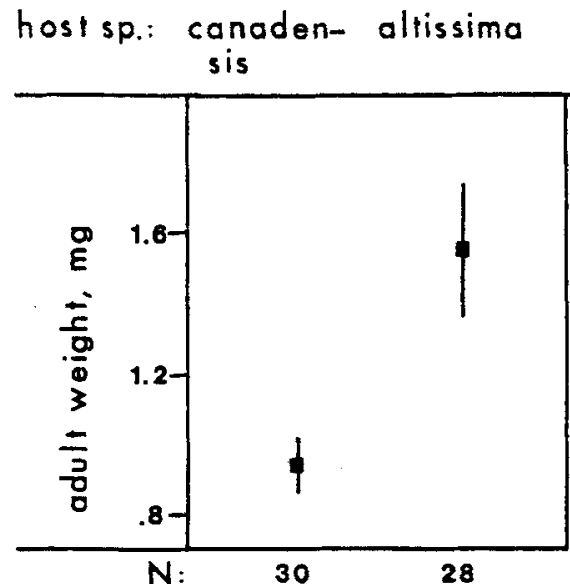

FIG. 2. Weights of wingless caligatum adults from naturally occurring colonies of S.canadensis and S.altissima (means and $95 \%$ confidence intervals).

one considers clones of all three species or restricts analysis to clones of canadensis. However, plant species sometimes seems to have an effect beyond that due to clonal differences. In particular, colonies on altissima had longer developmental times, larger adult weights, and larger total weight than those developing on canadensis. Weights of adults from naturally occurring colonies on canadensis and altissima (collected 10 August 1980, on the ESGR) provide evidence that the differences observed are not restricted to the caged colonies and apply to clones other than those selected for the cage experiments (Fig. 2).

An analysis of the differences in developmental times between $A C$ and $C P$ clones (cages paired within plant clones) reveals a significant interaction between aphid clone and plant species (Kruskal-Wallis, $P<0.01$ ). The significance of this result must be questioned, however, as the cages within a host species cannot be considered independent measures of performance, due to the low number of plant clones used. Had other plant clones been chosen there may have been no interaction between plant species and aphid clone. However, no significant interaction is observed when the non-parametric analysis is restricted to clones within plant species, an observation which supports the interpretation that plant species-aphid clone interaction is occurring.

The 1980 screenhouse colonies demonstrate a strong plant clone effect despite uniform growing conditions for plants and aphids (Fig. 3).

\section{Discussion}

The outstanding result is the strong effect of plant clone on aphid performance. Because hosts were physically separated in the field, differences could be attributed to: (i) microclimatic differences affecting the aphids directly, (ii) microhabitat differences affecting the plants, or (iii) genetic differences among the plant clones. All plants grew in full sun with similar densities of surrounding vegetation; microclimatic differences were probably as great among cages on the same clone as among clones. Also, one of the most pronounced differences in developmental times occurred between canadensis clones $1 \mathrm{C}$ and

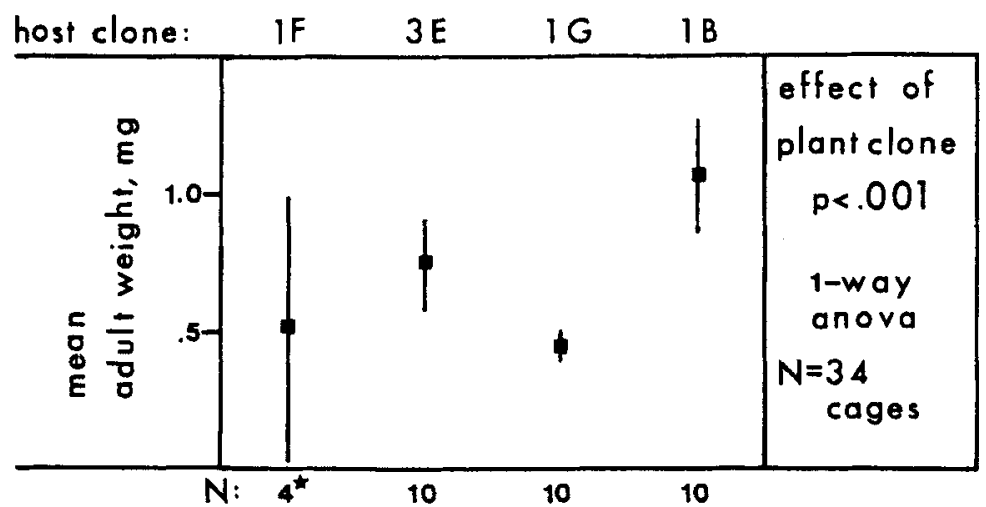

FIG. 3. Performance of one clone of $U$.callgatum on four clones of $S$.canadensis, as measured in a screenhouse. Means and $95 \%$ confidence intervals of cage means of adult weights. $*$ All aphids died in six of the ten colonies on $1 \mathrm{~F}$. 
$1 \mathrm{G}$ which grew less than $1 \mathrm{~m}$ apart. Thus genetic variation in quality of host clones seems the most likely source of the differences in aphid performance. The screenhouse experiments, by standardizing conditions for plant clones, provide more conclusive evidence for a genetic component of variation in host quality, whereas the field experiments have the advantage of demonstrating the occurrence of differences under natural conditions. Heterogeneity in host quality is of interest - at least to winged migrants colonizing new patches of host plant - whether it results from genetic or environmental factors.

The magnitude of the differences in host quality is substantial: there is more than a 2-fold difference between mean total colony weights attained on different clones of canadensis (e.g. IF v. 1G), and the difference between the two gigantea clones is even greater. If such differences were extended throughout the season, colonies on the higher quality host clones would experience a 30 - to 100 -fold larger increase in biomass. Several factors may prevent such differential rates of multiplication from being maintained. Colonies of caligatum rarely last more than 4-6 weeks on the same host patch, and winged morphs maturing on good and bad hosts probably have similar chances of initiating colonies on high quality hosts. Also, it is possible that relative quality of host clones depends on phenological state and thus may reverse itself during the season.

Clearly, migrants would benefit from the ability to discriminate between high and low quality clones within a host species. Thus, even for a specialist aphid like U.caligatum a condition which is actually typical in aphids (Eastop, 1972) - one might expect host selection to utilize plant characteristics beyond those sufficient to identify taxon.

Adult weight and total colony weight appear to be almost equivalent measures of performance $\quad(r=0.85,95 \%$ confidence interval: $0.79-0.90$ ) allowing tenative comparison of 1979 and 1980 results. The difference in order of host quality (1979: 1G > $3 E, 1 B ; 1980: 1 B, 3 E>1 G)$ most likely results from different responses of host genotypes to different environmental conditions (field $v$. pots in screenhouse).

Strong differences among clones of $U$.cali- gatum cannot be ruled out when only two have been compared. Much more thorough surveys of non-pest insects are required to determine if such species often exhibit the sort of variability in host relations that has been demonstrated in numerous crop-feeders, e.g. the aphids Amphorophora rubi (Briggs, 1965), Brevicoryne brassicae (Dunn \& Kempton, 1972) and Therioaphis maculata (Nielson \& Don, 1974), and the Hessian fly Mayetiola destructor (Hatchett \& Gallun, 1970).

\section{Acknowledgments}

L. R. Kirkendall read the manuscript and made many helpful comments. Other advice was given by W. D. Hamilton, E. G. Voss verified identifications of Solidago specimens. The E. S. George Reserve Fellowship Fund, the Rackham School of Graduate Studies, and a National Science Foundation Graduate Fellowship provided financial support.

\section{References}

Blackman, R.L. (1979) Variation and stability in aphid clonal lineages. Biological Joumal of the Linnean Society, 11, 259-277.

Briggs, J.B. (1965) The distribution, abundance, and genetic relationships of four strains of the rubus aphid (Amphorophor rubi (Kalt.)) in relation to raspberry breeding. Joumal of Horticultural Science, 40, $109-117$.

Clarke, B. (1976) The ecological genetics of hostparasite relationships. Genetic Aspects of HostParasite Relationshps (ed. by A. E. R. Taylor and R. Muller), pp. 87-103. Blackwell Scientific Publications, Oxford.

Day, P.R. (1974) Genetics of Host-Parasite Interaction. Freeman, San Francisco.

Dunn, J.A. \& Kempton, D.P.H. (1972) Resistance to attack by Brevicoryne brassicae among plants of Brussels sprouts. Annals of Applied Biology, 72, $1-11$.

Eastop, V.F. (1972) Deductions from the present day hostplants of aphids and related insects. Insect-Plant Relationships (ed. by H. F. van Emden), pp. 157-178. Blackwell Scientific Publications, Oxford.

Eastop, V.F. (1973) Bioty pes of aphids. Bulletin of the Entomological Society of New Zealand, 2, $40-51$.

Edmunds, G.F. \& Alstad, D.N. (1978) Coevolution in insect herbivores and conifers. Science, 199, $941-945$. 
Gallun, R.L., Starks, K.J. \& Guthrie, W.D. (1975) Plant resistance to insects attacking cereals. Annual Review of Entomology, 20, 337-357.

Gleason, H.A. \& Cronquist, A. (1963) Manual of Vascular Plants of Northeastern United States and Adjacent Canada. Van Nostrand, Princeton.

Hamilton, W.D. (1980) Sex versus non-sex versus parasite. Oikos, 35, $282-290$.

Hat chett, J.H. \& Gallun, R.L. (1970) Genetics of the ability of the Hessian fly, Mayetiola destructor, to survive on wheats having different genes for resistance. Annals of the Entomological Society of America, 63, 1400-1407.

Journet, A.R.P. (1980) Intraspecific variation in food plant favourability to phytophagous insects: psyllids on Eucalyptus blakelyi M. Ecological Entomology, 5, 249-261.

Mitter, C., Futuyma, D.J., Schneider, J.C. \& Hare,
J.D. (1979) Genetic variation and host plant relations in a parthenogenetic moth. Evolution, 33, 777-790.

Nie Ison, M.W. \& Don, H. (1974) Interaction between biotypes of the spotted alfal fa aphid and resistance in alfalfa. Economic Entomology, 67, 386-370.

Pielou, E.C. (1974) Biogeographic range comparisons and ovidence of geographic váriation in host-parasite relationships. Ecology, 55, 13591367.

Richards, W.R. (1972) Review of the Solidagoinhabiting aphids in Canada with descriptions of three new species (Homoptera: Aphididae). Canadian Entomologist, 104, 1-34.

Sokal, R.R. \& Rohlf, F.J.(1969) Biometry. Freeman, San Francisco.

Accepted 8 February 1981 